\title{
ORTHO-POSITRONIUM LIFETIME AS A DETECTOR OF SPIN-CROSSOVER
}

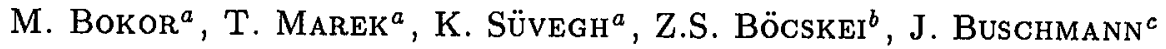 \\ AND A. VÉRTES ${ }^{a}$ \\ ${ }^{a}$ Department of Nuclear Chemistry, Eötvös Loránd University \\ P.O. Box 32, 1518 Budapest, Hungary \\ ${ }^{b}$ Chinoin Inc., Budapest, Hungary \\ 'Institute für Kristallographie, Freie Universität, Berlin, Germany
}

\begin{abstract}
Positron lifetime parameters were measured for the spin-crossover complexes $\left[\mathrm{Fe}(\mathrm{R}-1 H \text {-tetrazole })_{6}\right]\left(\mathrm{BF}_{4}\right)_{2}(\mathrm{R}=1$-ethyl, 1- $n$-propyl $)$ and for the diamagnetic $\left[\mathrm{Zn}(1-n \text {-propyl-1 } H \text {-tetrazole })_{6}\right]\left(\mathrm{BF}_{4}\right)_{2}$. Positronium forms with significant intensity in the studied compounds. The ortho-para conversion of ortho-positronium was used to follow the spin-crossover. Changes of the dynamic structure were found in the propyltetrazole complex between $150 \mathrm{~K}$ and $90 \mathrm{~K}$.
\end{abstract}

PACS numbers: $78.70 . \mathrm{Bj}, 36.10 . \mathrm{Dr}, 75.20 .-\mathrm{g}, 33.25 .+\mathrm{k}$

\section{Introduction}

ortho-Positronium (o-Ps) lifetime spectra can signal the spin-crossovers of transition metal ions in liquid [1] and solid phases [2]. The lifetime of $o$-Ps is shorter in the presence of unpaired electrons (high-spin state), due to the ortho-para conversion initiated by the paramagnetic spin-spin relaxation, and it is longer in the case of paired $3 d$ electrons (low-spin state), because of the lack of ortho-para conversion. The studied [ $\left.\mathrm{Fe}(1 \text {-alkyl- } 1 H \text {-tetrazole })_{6}\right]\left(\mathrm{BF}_{4}\right)_{2}$ compounds are octahedrally coordinated spin-crossover complexes of iron(II) - they undergo a temperature induced high-spin (HS) $\leftrightarrow$ low-spin (LS) transition

$$
t_{2 \mathrm{~g}}^{4} e_{\mathrm{g}}^{2} \underset{\text { hesting }}{\stackrel{\text { cooling }}{\rightleftarrows}} t_{2 \mathrm{~g}}^{6}, \quad{ }^{3} T_{2 \mathrm{~g}} \underset{\text { hesting }}{\stackrel{\text { cooling }}{\rightleftarrows}} A_{1 \mathrm{~g}}, \quad \Delta S=2 .
$$

The complexes are arranged into electrically neutral layers. There is a cleavage plane parallel to these planes and a pseudotrigonal symmetry axis perpendicular to each layer. In the ethyltetrazole complex (Fe-etz) only $2 / 3$ of the central Fe(II) ions undergo spin-crossover $\left(T_{1 / 2}=105 \mathrm{~K}\right)^{*}$, while the other $1 / 3$ remain in the HS state even at the lowest temperatures [3] while in the propyltetrazole complex (Fe-ptz) the spin-crossover $\left(T_{1 / 2}^{\uparrow}=135 \mathrm{~K}\right.$ and $T_{1 / 2}^{\downarrow}=128 \mathrm{~K}$, accompanied by a

${ }^{*}$ At $T_{1 / 2}$, the HS state fraction of ions undergoing the spin-crossover is equal to 0.5 . 
crystallographic phase transition at $130 \mathrm{~K}$ ) is practically complete [4]. The phase transition influences only the relative position of the complex layers. The partial spin-crossover of Fe-etz is traced back to the two inequivalent lattice sites in 2:1 proportion; the $\mathrm{Fe}(\mathrm{II})$ sites of $\mathrm{Fe}-\mathrm{ptz}$ are equivalent [3]. Fe-etz was studied to explore the effects of an incomplete spin-crossover on positron lifetime parameters. Average o-Ps lifetime $\left(\tau_{o-\mathrm{Ps}}\right)$ in Fe-ptz shows unexpected trend at $T>T_{1 / 2}$ which is also present in the isomorphous but diamagnetic Zn-ptz (Fig. 1a) [2]. Additional NMR spectroscopic analysis $\left({ }^{1} \mathrm{H},{ }^{11} \mathrm{~B}\right.$ and ${ }^{19} \mathrm{~F}$ spin-lattice relaxation time $\left(T_{1}\right)$ and spectrum measurements) [5] and X-ray diffraction measurements were carried out to reveal the underlying process.
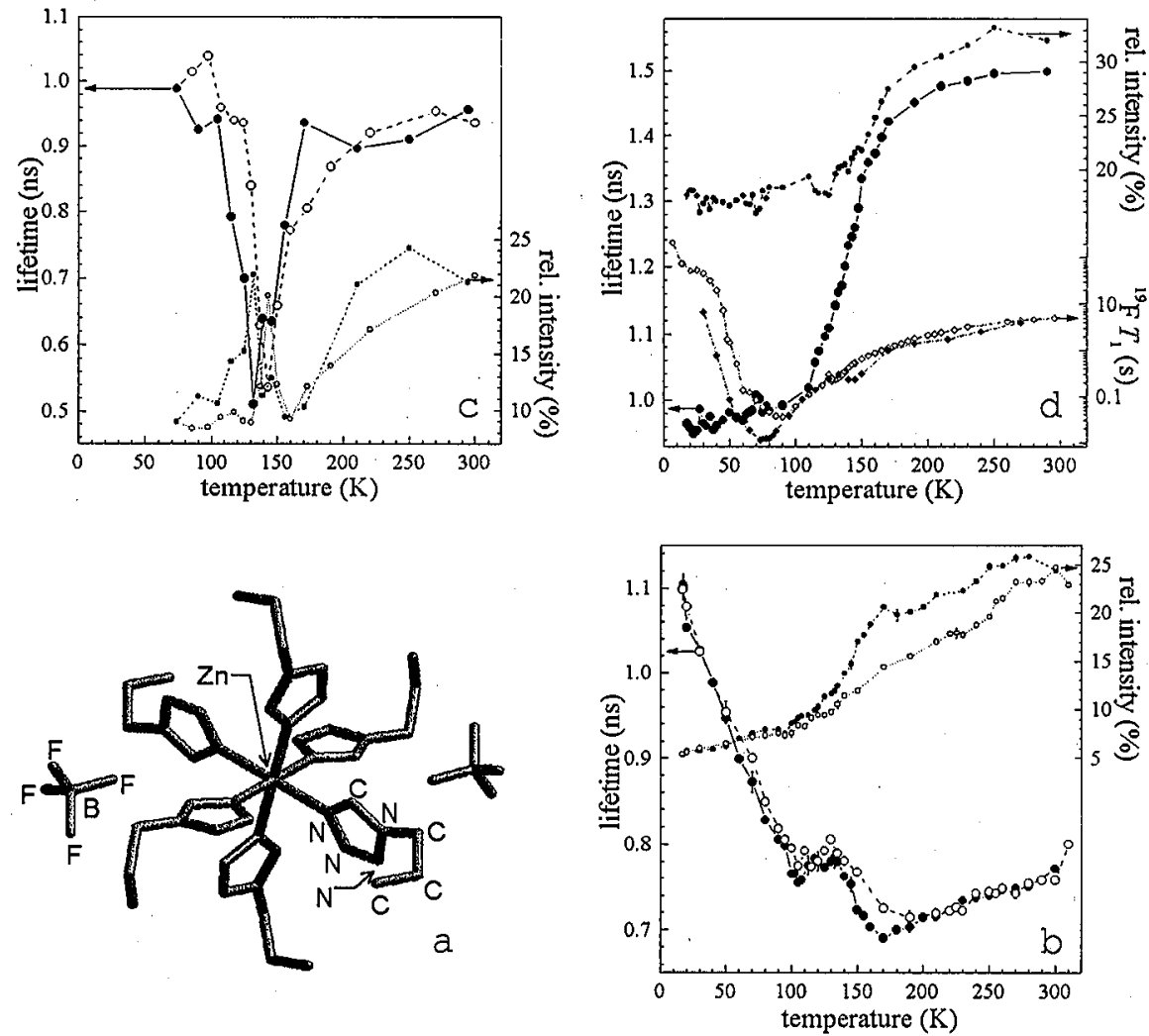

Fig. 1. (a) Single crystal X-ray structure of [ $\left.\mathrm{Zn}(1-n \text {-propyl- } H \text {-tetrazole })_{6}\right]\left(\mathrm{BF}_{4}\right)_{2}$ at $95 \mathrm{~K}$ (H-atoms are not shown). (b)-(d) o-Ps lifetime (big circles) and its relative intensity (small circles) for $\left[\mathrm{Fe}(1 \text {-ethyl- } 1 \mathrm{H} \text {-tetrazole })_{6}\right]\left(\mathrm{BF}_{4}\right)_{2}$, spin-crossover temperature $T_{1 / 2}=105 \mathrm{~K}(\mathrm{~b}) ;\left[\mathrm{Fe}(1-n \text {-propyl- } H \text {-tetrazole })_{6}\right]\left(\mathrm{BF}_{4}\right)_{2}, T_{1 / 2}^{\dagger}=135 \mathrm{~K}$ and $T_{1 / 2}^{\downarrow}=128 \mathrm{~K}$ (c); $\left[\mathrm{Zn}(1-n \text {-propyl-1H-tetrazole })_{6}\right]\left(\mathrm{BF}_{4}\right)_{2}$ (d). Solid symbols: cooling, open symbols: heating direction. Diamonds in graph (d): ${ }^{19} \mathrm{~F}$ spin-lattice relaxation time $\left(T_{1}\right)$ for [Zn(1- $n$-propyl- $1 \mathrm{H}$-tetrazole $\left.)_{6}\right]\left(\mathrm{BF}_{4}\right)_{2}$, solid symbols $\omega_{0}=29.0 \mathrm{MHz}$, open symbols $\omega_{0}=83.5 \mathrm{MHz}$; the minima indicate that $\mathrm{BF}_{4}^{-}$rotates above $40 \mathrm{~K}$, analysis of the 90 to $150 \mathrm{~K}$ temperature region suggests changing anion dynamics. 


\section{Experimental}

The alkyltetrazole ligands were prepared as described by Franke et al. [6] and the complexes themselves were prepared by the method of Poganiuch et al. [7]. The crude (polycrystalline) products were recrystallized from nitromethane to obtain single crystals. Single crystals were used to avoid the effects of grain boundaries on the positron-annihilation parameters.

The positron source was made by the deposition of ${ }^{22} \mathrm{NaCl}$ solution between two kapton foils. The source was placed between the pair of crystals and the source-sample sandwich was mounted in an evacuated cryostat. The pressure in the cryostat was kept at about $10^{-8}$ bar. The uncertainty of the temperature control was less than $0.1 \mathrm{~K}$. The heating or cooling rate between two measurements was about $1 \mathrm{~K} \mathrm{~min}^{-1}$. For the recording of lifetime spectra a fast-fast coincidence system was used. The spectra were collected in the 4096 channels of a multichannel analyser card with a $10 \mathrm{ps}$ channel time. The time resolution of the system was about $270 \mathrm{ps}$. The spectrum evaluation was carried out with the computer program RESOLUTION [8].

\section{Results and discussion \\ 3.1. $\left[\mathrm{Fe}(1 \text {-ethyl-1H-tetrazole })_{6}\right]\left(B F_{4}\right)_{2}$}

Fe-etz shows Ps formation (Fig. 1b) with a significant intensity both in the

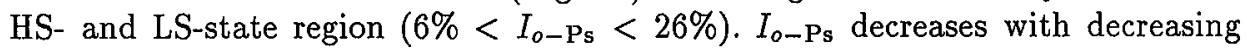
temperature throughout the whole temperature range, with two break-points: one at $170 \mathrm{~K}$ and the other at $T_{1 / 2}$. The most characteristic feature of the temperature dependence is the strong and monotonous increase in $\tau_{0-\mathrm{ms}}$ with the decrease in the temperature from the spin-crossover point (Fig. 1a). As the spin transition makes unpaired electrons disappear gradually upon cooling, the probability of the ortho-para conversion decreases and therefore $\tau_{o-P s}$ becomes longer. The residual $\mathrm{HS}$ state $\mathrm{Fe}$ (II) ions $\left(\gamma_{\mathrm{HS}}=1 / 3\right)$ induce ortho-para conversion even at temperatures well below $T_{1 / 2}$ and therefore $\tau_{0-\mathrm{Ps}}(T)$ changes gradually instead of producing a steplike trend in the vicinity of $T_{1 / 2}$ (see Fe-ptz, Fig. 1c).

\section{2. $\left[\mathrm{Fe}(1-n \text {-propyl-1H-tetrazole })_{6}\right]\left(\mathrm{BF}_{4}\right)_{2}$}

Ps forms (Fig. 1c) with high intensity also in Fe-ptz both in the HS- and LS-state region $\left(8 \%<I_{o-\mathrm{Ps}}<25 \%\right) . I_{o-\mathrm{Ps}}$ shows globally the same temperature dependence as in Fe-etz except the peaks at $T_{1 / 2}$ which can be attributed to the phase transition in the ptz complex. The $o$-Ps lifetime increases below $T_{1 / 2}$ (Fig. 1c). There is a pronounced step in $\tau_{o-P_{s}}$ of Fe-ptz between $T_{1 / 2}$ and $200 \mathrm{~K}$ where the $\tau_{o-\mathrm{Ps}_{\mathrm{s}}}(T)$ is controlled by the pick-off annihilation. The shape of the $\tau_{o-\mathrm{Ps}}(T)$ curves are the same in both $\mathrm{Zn}$-ptz. (Fig. 1d) and in Fe-ptz at $T>T_{1 / 2}(\mathrm{Fe}-\mathrm{ptz})$. The Fe-ptz and $\mathrm{Zn}$-ptz are isomorphous but there is no spin-crossover in Zn-ptz. Consequently, this lifetime trend between the $T_{1 / 2}$ and $200 \mathrm{~K}$ is not connected to the spin-crossover. (Probable underlying processes are discussed in Sec. 3.3.) The steplike increase in $\tau_{0-\mathrm{Ps}}$ below $T_{1 / 2}$ in Fe-ptz is a clear sign of the spin-crossover since the disappearing of $\mathrm{HS}$ state $\mathrm{Fe}(\mathrm{II})$ ions reduces the probability of ortho-para conversion resulting in longer $\tau_{o-\mathrm{Ps}}$. 


\section{3. $\left[Z n(1-n-p r o p y l-1 H \text {-tetrazole })_{6}\right]\left(B F_{4}\right)_{2}$}

$\tau_{o-P s}$ decreases by $30 \%$ when cooled from 200 to $90 \mathrm{~K}$ (Fig. 1d) and it is nearly constant at $T<90 \mathrm{~K}$. Comparing $\tau_{o-P s}(T>130 \mathrm{~K}$ ) for Fe-ptz (Fig. 1c) and $\mathrm{Zn-ptz} \mathrm{(Fig.} \mathrm{1d),} \mathrm{the} \mathrm{trends} \mathrm{are} \mathrm{the} \mathrm{same} \mathrm{and} \mathrm{the} \mathrm{difference} \mathrm{of} \mathrm{the} \mathrm{lifetime}$ values is explained by the presence of unpaired $e^{-}$spins in Fe-ptz. To explain $\tau_{o-P s}(T)$ for Zn-ptz above $90 \mathrm{~K}, \mathrm{NMR}$ spectroscopic [5] and X-ray diffraction methods were applied. ${ }^{1} \mathrm{H}$ and ${ }^{19} \mathrm{~F} T_{1}(T)$ (Fig. 1d) suggested that several changes of the dynamic structure - i.e. changes of the reorientational dynamics of propyl groups and anions - occur at $90 \mathrm{~K}<T<150 \mathrm{~K}$. The most likely sites where Ps exists are open spaces surrounded by the central ion, ligands, and anions. The observed decrease in $\tau_{o-P s}$ with decreasing temperature indicates an increase in the electron density in the surroundings of $o$-Ps. Possible origins of this change in the electron density are the following. The contraction of Ps sites caused by slight changes in the structure of the crystal - X-ray structures rule this out. Changes in the dynamics of propyl chains and anions result in changed electron density distribution and so, $\tau_{0-P s}$ is influenced - results of NMR analysis support this version.

\section{Conclusions}

$\tau_{o-P s}$ is strongly influenced by the abundance of unpaired electrons which can produce ortho-para conversion of the Ps. For the two investigated Fe-compounds it is understood that as the spin-crossover makes unpaired electrons disappear gradually as the sample is cooled down, the probability of the ortho-para conversion decreases and therefore $\tau_{o-P s}$ becomes longer. As a consequence, if there is a significant intensity of positronium, $\tau_{o-P s}$ detects sensitively the spin-crossover. The differences between the lifetime trends for the two compounds below $T_{1 / 2}$ can be explained by the effect of the residual HS state $\mathrm{Fe}$ (II) ions of Fe-etz. The increase in $\tau_{o-P s}$ in $\mathrm{Fe}$-ptz and $\mathrm{Zn}-\mathrm{ptz}$ above $130 \mathrm{~K}$ and $90 \mathrm{~K}$, respectively, can be explained by changes of the dynamic structure of the molecular groups in the vicinity of the Ps atoms.

\section{Acknowledgments}

This research was supported by the Hungarian National Science Fund (T014845), the Research and Development Grant of the Ministry of Culture and Education of Hungary (FKFP-0148/1997), and the Hungarian Academy of Sciences (AKP 97-33).

\section{References}

[1] A. Vértes, Zs. Kajcsos, L. Marczis, E. Brauer, J. Hüller, I. Zay, K. Burger, J. Phys. Chem. 88, 3969 (1984).

[2] A. Vértes, K. Süvegh, R. Hinek, P. Gütlich, J. Phys. Chem. Solids 55, 1269 (1994).

[3] R. Hinek, H. Spiering, D. Schollmeyer, P. Gütlich, A. Hauser, Chem. Eur. J. 2, 1427 (1996).

[4] L. Wiehl, Acta Crystallogr. B 49, 289 (1993). 
[5] (a) M. Bokor, T. Marek, K. Tompa, J. Magn. Reson. A 122, 157 (1996); (b) M. Bokor, T. Marek, K. Tompa, A. Vértes, J. Mol. Struct. 410-411, 1 (1997). [6] P.L. Franke, J.G. Haasnoot, A.P. Zuur, Inorg. Chim. Acta 59, 5 (1982).

[7] P. Poganiuch, S. Decurtins, P. Gütlich, J. Am. Chem. Soc. 112, 3270 (1990).

[8] P. Kirkegaard, M. Eldrup, O.E. Mogensen, N.J. Pedersen, Comput. Phys. Commun. 23, 307 (1981). 